\section{Technetium Tc99m-Tetrofosmin Brain Single-Photon Emission CT for the Diagnosis of Malignant Meningiomas}

We read with great interest a recent article by Nagar et $\mathrm{al}^{1}$ in the American Journal of Neuroradiology investigating the usefulness of diffusion-weighted MR imaging in differentiating atypical/malignant meningiomas from benign meningiomas and detecting histologic dedifferentiation to higher tumor grade. The authors studied 48 cases of meningiomas and found that the mean apparent diffusion coefficient and normalized apparent diffusion coefficient ratios of atypical/ malignant meningiomas were significantly lower compared with benign meningiomas. The mean apparent diffusion coefficient and normalized apparent diffusion coefficient cutoff threshold values representatively discriminating the 2 clinical entities was $0.80 \times 10(-3)$ $\mathrm{mm}^{2} / \mathrm{s}$ and 0.99 , respectively, as indicated by the receiver operating characteristic curve analysis. Finally, diffusion-weighted MR imaging gave promise in the detection of meningioma histologic dedifferentiation to higher tumor grade. ${ }^{1}$

Our ongoing research deals with the usefulness of functional brain tumor imaging by single-photon emission CT (SPECT). We currently investigate the imaging properties of technetium-Tc $99 \mathrm{~m}$-tetrofosmin ( $\left.{ }^{99 \mathrm{~m}} \mathrm{Tc}-\mathrm{TF}\right)$, a lipophilic diphosphine routinely used for myocardial perfusion imaging that also displays tumor-seeking properties. We evaluated prospectively whether ${ }^{99 \mathrm{~m}} \mathrm{Tc}-\mathrm{TF}$ uptake correlates with the cellular proliferative activity of the meningioma (as assessed by Ki-67 index and flow cytometry) and grade. We found that ${ }^{99 \mathrm{~m}} \mathrm{Tc}-\mathrm{TF}$ SPECT can reliably distinguish typical meningiomas from malignant meningiomas. A significant correlation of radiotracer uptake with both Ki-67 expression and the percentage of the cell fraction on $\mathrm{S}$ phase and the level of aneuploidy of meningiomas ${ }^{2,3}$ was also detected. Furthermore, there was a significant correlation between the intensity of tracer uptake and tumor recurrence 1 year postoperatively. ${ }^{2}$

Meningiomas constitute approximately $20 \%$ of all intracranial tumors, and though they usually have a relatively benign clinical course and only a $15 \%$ recurrence rate, atypical and malignant meningiomas are associated with high recurrence rates and poor outcome. ${ }^{4}$ Apart from tumor grade, the Ki-67 index and status of DNA ploidy and S phase (as determined by flow cytometry) have been directly correlated with the biologic behavior of the meningioma. ${ }^{4,5}$ Because ${ }^{99 \mathrm{~m}} \mathrm{Tc}$-TF brain SPECT has a favorable cost and is widely available, we propose this imaging technique as an alternative method for the differentiation of typical from malignant meningiomas that, in addition, provides a noninvasive estimation of the proliferative potential of meningiomas.

\section{References}

1. Nagar VA, Ye JR, Ng WH, et al. Diffusion-weighted MR imaging: diagnosing atypical or malignant meningiomas and detecting tumor dedifferentiation. AJNR Am J Neuroradiol 2008;29:1147-52

2. Fotopoulos AD, Alexiou GA, Goussia A, et al. (99m)Tc-Tetrofosmin brain SPECT in the assessment of meningiomas-correlation with histological grade and proliferation index. J Neurooncol 2008;89:225-30

3. Alexiou GA, Vartholomatos G, Tsiouris S, et al. Evaluation of meningioma aggressiveness with ${ }^{{ }^{99} \mathrm{~m}}$ Tc-Tetrofosmin SPECT. Clin Neurol Neurosurg 2008;110:645-48

4. Kasuya $\mathrm{H}$, Kubo $\mathrm{O}$, Tanaka $\mathrm{M}$, et al. Clinical and radiological features related to the growth potential of meningioma. Neurosurg Rev 2006;29:293-96

5. Zellner A, Meixensberger J, Roggendorf W, et al. DNA ploidy and cell-cycle analysis in intracranial meningiomas and hemangiopericytomas: a study with high-resolution DNA flow cytometry. Int J Cancer 1998;79:116-20

G.A. Alexiou

S. Voulgaris

Department of Neurosurgery

S. Tsiouris

A.D. Fotopoulos

Department of Nuclear Medicine

A.P. Kyritsis

Department of Neurology and Neurosurgical Institute

K.S. Polyzoidis

Department of Neurosurgery and Neurosurgical Institute

University Hospital of Ioannina

Ioannina, Greece

DOI 10.3174/ajnr.A1286 